\title{
Lattice Considerations for a Tau-Charm Factory*
}

\author{
L. C. Teng and E. A. Crosbie \\ Advanced Photon Source, Argonne National Laboratory \\ 9700 South Cass Avenue, Argonne, Illinois 60439 USA

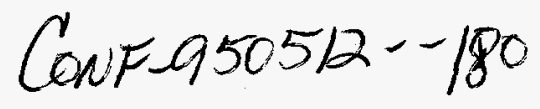

\section{Abstract}

The magnet lattice appropriate for a tau-charm factory had been studied extensively [1]. Here we discuss two possible simplifying features which make the design, construction, and operation of the machine simpler without sacrificing performance. These two features may be characterized and identified as a) luminosity optimization in the "monochromatic" mode, and b) chromaticity correction with sextupoles only in the arcs.

\section{INTRODUCTION}

A tau-charm factory is a high luminosity $\left(L \geq 10^{33} \mathrm{~cm}^{-2} \mathrm{~s}^{-1}\right)$ $e^{+} e^{-}$collider with a center-of-mass energy, $2 E$ ( $E=$ beam energy), adjustable from 3 to $6 \mathrm{GeV}$. The lower limit is set by the charmonium, $\mathrm{J} / \psi$, production threshold of $3.1 \mathrm{GeV}$ and the upper limit is set by the production threshold for pairs of charmed baryons, e.g. at $2 \times 2.74 \mathrm{GeV}$ for $\left(\Omega_{c}^{-}, \Omega_{c}^{-}\right)$. The high luminosity requires that the two beams be stored in separate rings, here assumed to be located one directly above the other. Because of the extremely narrow width of $J / \psi \quad \Gamma=$ $0.086 \mathrm{MeV}$ ), it has been proposed [1] that for $\mathrm{J} / \Psi$ production the lattices of the two rings be tuned to have large equal and opposite vertical dispersion functions, $\pm D_{y}$, for the two beams at the collision point. The collision energy spread would then arise only from the vertical betatron width $\sigma_{\mathrm{y} \beta}$ of the beams. To keep the energy spread within the $J / \psi$ width, we must have

$$
\sigma_{y \beta} \leq\left(\frac{\Gamma}{2 E}\right) D_{y}
$$

This so-called "monochromatic" tuning was considered necessary for $\mathrm{J} / \Psi$ production even at a sacrifice of luminosity. We will show that, properly optimized, the achievable luminosity is actually higher for the "monochromatic" tune.

\section{Optimization of LUMINOSITY}

For the head-on collision of two identical beam bunches containing $\mathrm{N}$ particles each, the beam-beam tune shifts are

$$
\left\{\begin{array}{l}
\xi_{x}=N K \frac{\beta_{x}}{\sigma_{x}\left(\sigma_{x}+\sigma_{y}\right)}, \\
\xi_{y}=N K \frac{\beta_{y}}{\sigma_{y}\left(\sigma_{x}+\sigma_{y}\right)},
\end{array}\right.
$$

* Work supported by the U.S. Department of Energy, Office of Basic Energy Sciences, under Contract No. W-31-109-ENG-38. where $\mathrm{K} \equiv \mathrm{r} / 2 \pi \gamma=1.48 \times 10^{-19} \mathrm{~m}$ at the beam energy of $\mathrm{E}=$ $1.55 \mathrm{GeV}$ for $\mathrm{J} / \Psi$ production. In these equations and throughout this paper all symbols have their conventional meanings and all quantities are evaluated at the collision point. The luminosity is

$$
L=\frac{f}{4 \pi} \frac{N^{2}}{\sigma_{x} \sigma_{y}},
$$

where $f$ is the bunch collision frequency. The luminosity is clearly largest when $\xi_{\mathrm{x}}=\xi_{\mathrm{y}} \equiv \xi=$ empirical maximum attainable value $\cong 0.04$. This condition gives

$$
\frac{\beta_{y}}{\sigma_{y}}=\frac{\beta_{x}}{\sigma_{x}}
$$

When this condition is satisfied, we have

$$
N=\frac{\xi}{K} \frac{\sigma_{x}^{2}}{\beta_{x}}\left(1+\frac{\beta_{y}}{\beta_{x}}\right)
$$

and

$$
\mathrm{L}=\frac{\mathrm{f}}{4 \pi} \frac{\mathrm{N}^{2}}{\beta_{\mathrm{y}}} \frac{\beta_{\mathrm{x}}}{\sigma_{\mathrm{x}}^{2}} .
$$

For the standard tuning $D_{x}=D_{y}=0$ and Eq. (4) gives $\beta_{y} / \beta_{x}=\varepsilon_{y} / \varepsilon_{x}=a$ value generally much less than unity. In this case, we should take $\beta_{y}=0.01 \mathrm{~m}=$ the smallest practicable value in one of the two dimensions. Taking further, $f=$ $30 \mathrm{MHz}\left(10-\mathrm{m}\right.$ bunch spacing) and $\varepsilon_{\mathrm{x}}=250 \times 10^{-9} \mathrm{~m}$-rad we obtain

\begin{tabular}{cccc}
$\beta_{\mathrm{x}}(\mathrm{m})$ & $\beta_{\mathrm{y}} / \beta_{\mathrm{x}}$ & $\mathrm{N}$ & $\mathrm{L}\left(\mathrm{cm}^{-2} \mathrm{~s}^{-1}\right)$ \\
\hline 0.02 & 0.5 & $1.02 \times 10^{11}$ & $0.98 \times 10^{33}$ \\
0.05 & 0.2 & $0.81 \times 10^{11}$ & $0.63 \times 10^{33}$
\end{tabular}

This shows that with these parameters one can barely reach the desired luminosity of $10^{33} \mathrm{~cm}^{-2} \mathrm{~s}^{-1}$.

For the "monochromatic" tuning, the example lattice we studied gives $D_{\mathrm{y}}=0.4 \mathrm{~m}$ and $\sigma_{\mathrm{E}} \cong 0.6 \mathrm{MeV}$ at the beam energy $\mathrm{E}=1.55 \mathrm{GeV}$ for $\mathrm{J} / \psi$. Equations (1) and (4) then give

$$
\sqrt{\beta_{\mathrm{y}} \varepsilon_{\mathrm{y}}}=\frac{\Gamma}{2 \mathrm{E}} \mathrm{D}_{\mathrm{y}} \cong 11 \mu \mathrm{m}
$$

and 


\section{DISCLAIMER}

This report was prepared as an account of work sponsored by an agency of the United States Government. Neither the United States Government nor any agency thereof, nor any of their employees, makes any warranty, express or implied, or assumes any legal liability or responsibility for the accuracy, completeness, or usefulness of any information, apparatus, product, or process disclosed, or represents that its use would not infringe privately owned rights. Reference herein to any specific commercial product, process, or service by trade name, trademark, manufacturer, or otherwise does not necessarily constitute or imply its endorsement, recommendation, or favoring by the United States Government or any agency thereof. The views and opinions of authors expressed herein do not necessarily state or reflect those of the United States Government or any agency thereof. 


\section{DISCLAIMER}

Portions of this document may be illegible in electronic image products. Images are produced from the best available original document. 


$$
\frac{\beta_{y}}{\varepsilon_{y}}=\left[1+\left(\frac{2 \sigma_{E}}{\Gamma}\right)^{2}\right] \frac{\beta_{x}}{\varepsilon_{x}} \cong 190 \frac{\beta_{x}}{\varepsilon_{x}} .
$$

It is unlikely that $\varepsilon_{y}$ can be made smaller than $\varepsilon_{x} / 190$. Equation (8) then says that $\beta_{x}$ must be smaller than $\beta_{y}$ We therefore set $\beta_{x}$ equal to the minimum practical value of $0.01 \mathrm{~m}$. Equations (7) and (8) then give

$$
\beta_{y} \equiv 0.03 \mathrm{~m} \text { and } \varepsilon_{\mathrm{y}} \equiv 4 \times 10^{-9} \mathrm{~m} \text {, }
$$

and Eqs. (5) and (6) give

$$
\mathrm{N} \cong 2.7 \times 10^{11} \text { and } \mathrm{L} \cong 2.3 \times 10^{33} \mathrm{~cm}^{-2} \mathrm{~s}^{-1} \text {. }
$$

The higher luminosity is derived, of course, from the higher beam intensity which is, however, allowed by the beam-beam effect for this design.

The very small collision energy spread is desirable at all energies and for all experiments. There is no need ever for the zero-dispersion design.

\section{ARC LATTICE}

The linear lattice is conventional and consists of two $180^{\circ}$ anc sections joined by two $118.4-\mathrm{m}$-long straight sections: one for injection and $\mathrm{rf}$, the other for beam collision and the detector assembly. The arc sections are composed of ten $60^{\circ} 7.4-\mathrm{m}$ FODO cells each (including the horizontal dispersion suppressor cells) with parameters adjusted to give a natural horizontal emittance of $-250 \times 10^{-9} \mathrm{~m}$.

\section{Colliding Straight LatTice}

The injection straight lattice is conventional and straightforward and will, therefore, not be discussed. The collision or interaction straight lattice is developed along the following guiding features:

1. The collision is head-on. The strong low- $\beta$ quadrupole doublets next to the collision point are superconducting, and are common to and have identical focal actions on both beams. The lattice is, thus, symmetric. Outboard of the common quadrupole doublets, the beams are separated vertically, first by electrostatic separators followed by septum dipoles. The linear orbit functions are adjusted to $D_{x}=0, D_{y}= \pm 0.4 \mathrm{~m}, \beta_{x}=0.01 \mathrm{~m}, \beta_{y}=0.03 \mathrm{~m}, \varepsilon_{y}<$ $4 \times 10^{-9} \mathrm{~m}$ and, since the lattice is symmetric, $D_{x}^{\prime}=D_{y}^{\prime}=$ $\alpha_{x}=\alpha_{y}=0$. (Here, as before, all values given are those at the collision point.)

2. We choose not to cross the beams so that a) the central collision energy can be fine-adjusted (up to $\sim 2 \sigma_{\mathrm{E}} \cong 1.2 \mathrm{MeV}$ ) by vertically parallel-displacing one beam against the other, and that b) the no crossing geometry in the injection straight is more convenient for injection and for accommodating if cavities. The design of the matched linear lat- tice offers essentially no special difficulties and is shown in Fig. 1. However, some special attention is required for chromaticity correction and dynamic aperture problems.

\section{CHROMATICITY CORRECTION AND DYNAMIC APERTURES}

Without correction the natural chromaticities of the linear lattice are approximately -27 (horizontal) and -39 (vertical). The major contribution comes from the very strong superconducting low- $\beta$ quadrupoles. The traditional wisdom is to correct the chromaticity "at the source." To do this, many additional complicated and difficult matching conditions must be met in the linear straight lattice

For this tau-charm factory we tried to avoid this complication by locating the sextupoles in the arcs only. Since the arcs consist of $60^{\circ}$ FODO cells, we can place two pairs of sextupoles in each arc such that the two members in each pair are identical in strength, located at identical $\beta$ values, and separated by $\pi$ phase-advance. In this way, the chromaticity correcting effects of the two members add, but their resonance driving effects cancel. With one pair placed at high $\beta_{\mathrm{x}}$ and the other at high $\beta_{y}$, we can adjust the strengths of the two pairs to correct the $x$ - and $y$-chromaticities simultaneously. We found, indeed, that after correcting the chromaticities to zero, the dynamic apertures are $>80 \sigma\left(\sigma=\right.$ larger of $\sigma_{x}$ and $\left.\sigma_{y}\right)$ at the central energy and $>25 \sigma$ at $\pm 10 \sigma_{\mathrm{E}}$ from the central energy.

Actually, with the rather large vertical dispersions at the superconducting low $-\beta$ quadrupoles, one can provide chromaticity corrections "at the source" by inserting superconducting skew-sextupole coils in these low- $\beta$ quadrupoles, except it is now impossible to cancel their resonance-driving harmonics by other skew-sextupoles located $\pi$-phase advance away.

\section{REFERENCE}

[1] J. M. Jowett, Frontiers of Particle Beams: Factories with e+e- Rings, edited by M. Dienes, M. Month, B. Strasser, S. Turner (Springer Verlag, 1994); and the many references given in this paper. 


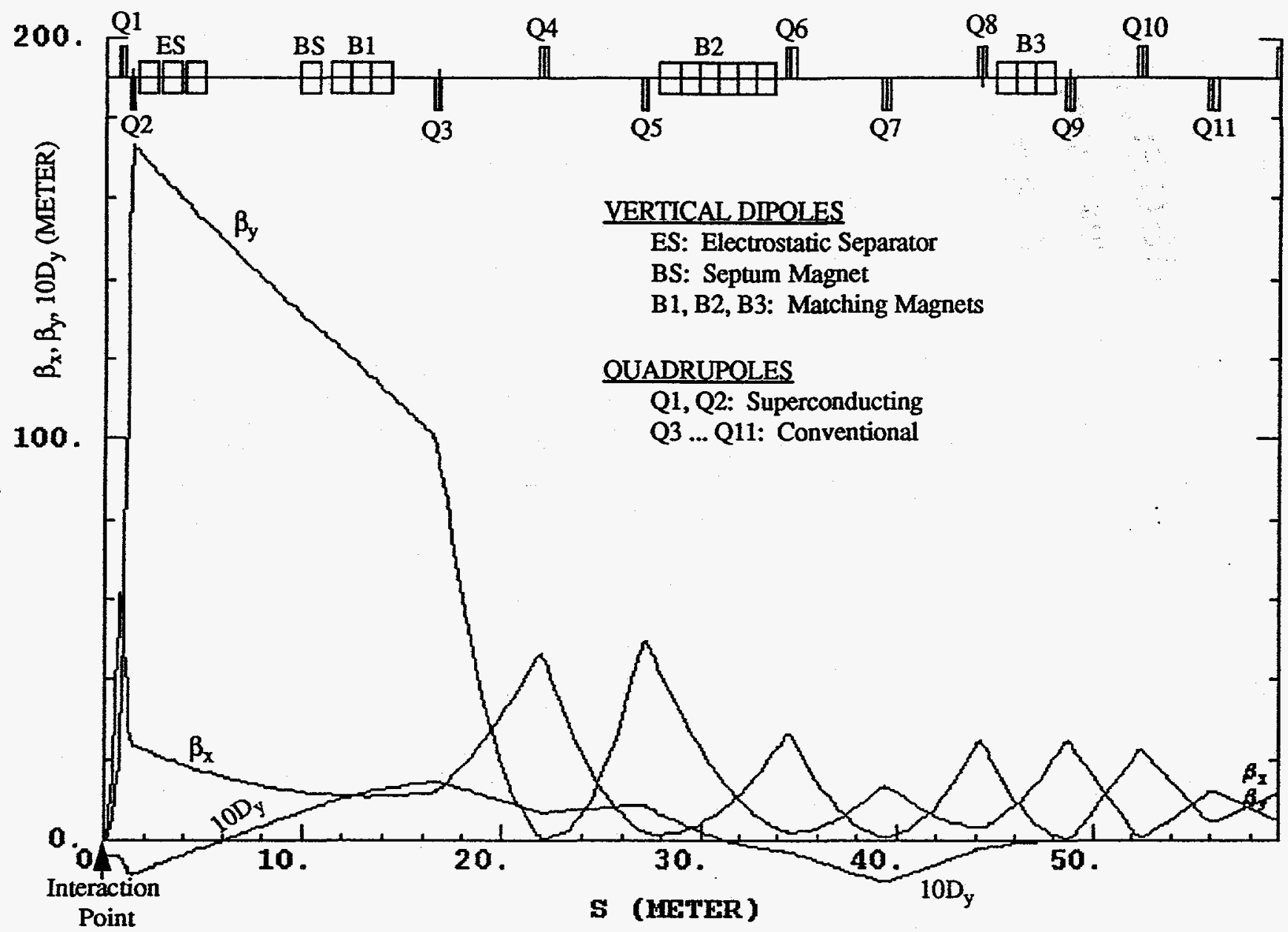

Figure 1: Linear orbit functions across one-half of the interaction straight section. At the collision point $\beta_{x}=1 \mathrm{~cm}, \beta_{y}=3 \mathrm{~cm}, D_{x}=0, D_{y}=0.4 \mathrm{~m}$.

For the complete ring, $\varepsilon_{\mathrm{x}}=252 \mathrm{~nm}, \varepsilon_{\mathrm{y}}=3.8 \mathrm{~nm}$, and $\sigma_{\mathrm{E}} / \mathrm{E}=0.38 \times 10^{-3}$.

The vertical separation of the rings is $\sim 1.23 \mathrm{~m}$. 